\title{
Homozygous intronic mutation leading to inefficient transcription combined with a novel frameshift mutation in F13A1 gene causes FXIII deficiency
}

\author{
Wei Wang ${ }^{1,2,8}$, Lijun Huang ${ }^{1,3,8}$, Qiuling $\mathrm{Ma}^{1,4}$, Deqian Xiao ${ }^{1,5}$, Xiaodan Chen ${ }^{6}$, Zhigang Yang ${ }^{4}$, \\ Xiaoying Wang ${ }^{1}$, Keyuan Zhou ${ }^{5}$, Gang $\mathrm{Li}^{3}$, Man Xiao ${ }^{1}$, Guankui $\mathrm{Du}^{1}$, Xinbao $\mathrm{Hao}^{7}$ and Wangwei Cai ${ }^{1}$
}

Two novel mutations, 602-605delAAAG in exon 5 and $\operatorname{Int1}(+12) C>A$, of the $F 13 A 1$ gene were identified in a Chinese factor XIII (FXIII)-deficient family. The 602-605delAAAG mutation results in the premature termination of translation. To determine the functional effect of the Int1(+12)A mutation, we transiently expressed luciferase reporters in U937 cells. We found that the first 951 bp of F13A1 intron 1 is involved in regulating the expression of the F13A1 gene and that Int1(+12)A results in its reduced expression. Electrophoretic mobility shift assay indicated that Int1(+12)A causes reduced protein binding. An Sp1 site was predicted in the sequence containing Int1(+12)C, which the Int1(+12)A mutation eliminates. Co-transfection of a plasmid expressing Sp1 revealed that Sp1 is involved in regulating the expression of FXIIIA and that Int1(+12)A leads to inefficient transcription. These results provide the first insight into a novel regulatory mechanism involving intron 1 in the F13A1 gene. Journal of Human Genetics (2011) 56, 460-463; doi:10.1038/jhg.2011.41; published online 21 April 2011

Keywords: F13A1 gene mutation; FXIII deficiency; Frameshift mutation; intronic mutation; intronic Sp1-binding site

\section{INTRODUCTION}

Inherited deficiency of FXIII is a rare, autosomal recessive disorder, leading to life-long bleeding diathesis and defective wound healing in affected patients, and a high risk of spontaneous abortion in affected women..$^{1-2}$ Mutations in the A or B subunit gene are responsible for the disorder. ${ }^{1-7}$ The disorder usually results from mutations in subunit A, but is occasionally attributable to mutations in subunit B (http:// www.hgmd.cf.ac.uk/ and http://www.f13-database.de). We describe two novel mutations in a Chinese FXIII-deficient family and characterize a novel mechanism involved in regulating the expression of the F13A1 gene.

\section{MATERIALS AND METHODS}

The proband was female, born in 1983 at full term following a normal delivery. She had umbilical cord bleeding a few days after birth and recurrent posttraumatic subcutaneous bleeding episodes. Her younger brother displayed a slight bleeding disorder. Other family members had no bleeding tendency. The proband was diagnosed using a clot stability test in $5 \mathrm{M}$ urea. Informed consent was obtained from all family members in accordance with the Declaration of Helsinki, and the study was approved by the ethical review committee of the Affiliated Hospital of Hainan Medical College. FXIII activity and plasma FXIIIA antigen were determined as described previously, using fibrinogen and 5-biotinamido)pentylamine as substrates ${ }^{8}$ and an enzyme-linked immunosorbent assay, respectively (Supplementary materials).

Genomic DNA was extracted from leukocytes. Exons 1-15 of the F13A1 gene were amplified by PCR (Supplementary Table 1). Mutations were screened by single-stranded conformation polymorphism analysis and confirmed by DNA sequencing. Total RNA was extracted from lymphocytes and reverse transcribed with the SuperScript II reverse transcription (RT) kit (Invitrogen Life Technologies, Carlsbad, CA, USA). The complementary DNA generated was amplified, using $\beta$-actin as the internal control.

A PCR-based strategy was used to generate constructs containing $114 \mathrm{bp}$ of the minimum promoter, ${ }^{9}$ exon 1 and $3^{\prime}$ serial deletions of intron 1 from nt +951 (Figure 1a and Supplementary materials). The Int1+12A mutation was introduced into the construct using site-directed mutagenesis. The sequences of all reporter constructs were verified by DNA sequencing. The constructs and pGL3Basic, used as the negative control, were transfected into cultured cells with Lipofectamine 2000 (Invitrogen). The cells were harvested $48 \mathrm{~h}$ after transfection and cell lysates were prepared. Reporter gene activities were determined using the Dual-Luciferase Reporter Assay System (Promega, Madison, WI, USA). Luciferase intensities were normalized to the internal control (SV40-Renilla) to assess transfection efficiency. All experiments were repeated at least three times using three different preparations of each construct tested. Plasmid pN3-Sp1 (Invitrogen), which expresses Sp1, was co-transfected into U937 cells with a construct with or without the Int1+12A mutation.

${ }^{1}$ Department of Biochemistry, Hainan Medical College, Haikou, Hainan, PR China; ${ }^{2}$ The Clinical Hematology Laboratory, Faculty of Medical Laboratory Science, Guangdong Medical College, Zhanijiang, Guangdong, PR China; ${ }^{3}$ Department of Biochemistry and Molecular Biology, Peking University Health Science Center, Beiijing, PR China; ${ }^{4}$ Department of Hematology, Affiliated Hospital of Guangdong Medical College, Zhanjiang, Guangdong, PR China; ${ }^{5}$ Department of Biochemistry, Guangdong Medical College, Zhanjiang, Guangdong, PR China; ${ }^{6}$ The Clinical Laboratory, Affiliated Hospital of Hainan Medical College, Haikou, Hainan, PR China and ${ }^{7}$ Department of Hematology, Affiliated Hospital of Hainan Medical College, Haikou, Hainan, PR China

${ }^{8}$ These authors contributed equally to this work.

Correspondence: Dr W Cai, Department of Biochemistry, Hainan Medical College, Chengxi Road No. 3, Haikou, Hainan 571101, PR China.

E-mail: caiww591020@163.com

Received 17 November 2010; revised 14 March 2011; accepted 16 March 2011; published online 21 April 2011 
Nuclear extracts were prepared from U937 cells. Double-stranded oligonucleotide probes (Supplementary Table 2), binding the region from -4 to +26 bp, with nucleotide $\mathrm{C}$ or A at position +12 in intron 1, were end-labeled with $\gamma-{ }^{32} \mathrm{P}$-ATP. Binding reactions were performed as described in Supplementary materials. In competition experiments, a 125-fold molar excess of unlabeled probe was included. The DNA-protein complexes were separated on $8 \%$ polyacrylamide gels, which were dried under vacuum and exposed to X-ray film.

\section{RESULTS AND DISCUSSION}

Assays of FXIII activity and plasma FXIIIA antigen in the patients and family members revealed very low relative levels of FXIII activity and FXIIIA antigen in the patients (Supplementary Table 3). These data confirmed the previous diagnosis of FXIII deficiency made with the urea solubility test.

Single-stranded conformation polymorphism analysis revealed band shifts in the amplification products of exon 1 (Supplementary Figure 1) and exon 5 (Supplementary Figure 1), indicating mutations in these exons. No abnormal band shift was observed in 120 healthy individuals, confirming the band shifts in exons 1 and 5 of the patients as causative. Therefore, the abnormal band shifts in exons 1 and 5 are probably associated with the patient's FXIII deficiency.

Sequencing analysis revealed that the band shift in exon 1 resulted from a C-to-A transversion at position +12 in intron 1 $(\operatorname{Int} 1(+12) \mathrm{C}>\mathrm{A})$. The proband and her younger brother were homozygous for the $\operatorname{Int} 1(+12) \mathrm{C}>\mathrm{A}$ mutation, whereas her mother and father were heterozygous for the Int $1(+12) \mathrm{C}>\mathrm{A}$ mutation (Supplementary Figure 2). However, the variation is polymorphic in African and European descents (http://pga.gs.washington.edu), which may be due to racial differences. Indeed, difference in allelic frequency, such as F13A Val34Leu polymorphism, had been observed between Asian and other populations. ${ }^{10,11}$

Direct sequencing of the exon 5 PCR fragment revealed two separate sequences (Supplementary Figure 3), suggesting a deletion or insertion in one of the alleles. Subcloning and sequencing revealed a deletion of AAAG at 602-605 (602-605delAAAG) or AAGA at 603-606 in the GAGAAAGAAAGAGAAGAG region, leading to a predicted truncated peptide of 204 amino acids (Supplementary Figure 3). There are no other mutations in F13A1 gene. These results confirm that the two mutations are responsible for the FXIII deficiency in the patients. A pedigree analysis of the family is shown in Supplementary Figure 4.

Previous studies of other genes have shown that intronic sequences are involved in regulating gene expression. ${ }^{12-16}$ In this study, reduced levels of FXIIIA antigen and mRNA were observed in the patients (Supplementary Table 3 and Supplementary Figure 5), suggesting that the Int $1(+12) \mathrm{C}>\mathrm{A}$ mutation affects the expression of the F13A1 gene. To determine the functional effects of the $\operatorname{Int} 1(+12) \mathrm{C}>\mathrm{A}$ mutation in the $F 13 A 1$ gene, luciferase reporters were transiently expressed in U937 cells. The minimal F13A1 promoter plus exon 1 and the first $951 \mathrm{bp}$ of intron 1 correctly transcribed a luciferase reporter gene (Figure 1b). Serial 3' deletion analysis revealed that deletions from +951 to $+610,+351$ and +91 resulted in increased luciferase activity, whereas deletions from +951 to +227 and +54 resulted in dramatically reduced luciferase activity (Figure 1b), suggesting that the first 951 bp of intron 1 are involved in regulating F13A1 gene expression. Because the deletion from +951 to +91 (construct pF13AInt+91) displayed the highest luciferase activity, Int $1(+12) \mathrm{A}$ was introduced into pF13AInt+91 to evaluate its effect. The luciferase activity in cells transfected with mutant construct pF13AInt+91mt12 was significantly reduced compared with that in cells transfected with construct pF13AInt+91 (Figure 1b). This is consistent with the reduced FXIIIA antigen levels and reduced mRNA levels observed. These results strongly suggest that the $\operatorname{Int} 1(+12) \mathrm{C}>\mathrm{A}$ mutation is a transcription-affecting mutation associated with FXIII deficiency.

Prediction of the relevant cis element in the first $951 \mathrm{bp}$ of intron 1 of the F13A1 gene with MatInspector (http://www.genomatix.de) identified several putative Spl-binding sites in the intron 1 region (Figure 2a). The Int1(+12)A mutation eliminates the Sp1-binding site between base pairs +8 and +15 of intron 1 , indicating that this site might be involved in transcription. An electrophoretic mobility shift assay revealed a single complex formed by both the wild-type probe and mutated probe. However, the band representing the complex formed with the mutated probe was markedly weaker (Figure $2 b$ ).

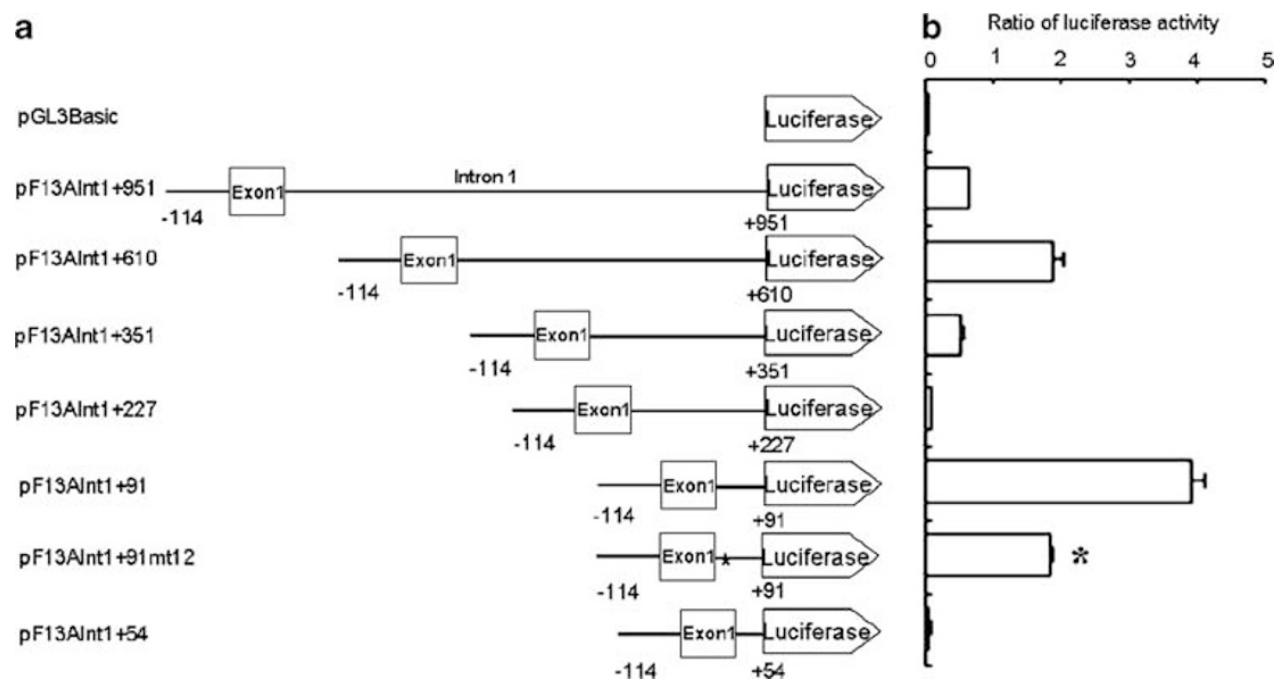

Figure 1 Functional characterization of the sequence of intron 1 of the $F 13 A 1$ gene. (a) The diagram shows various fragments created by serial $3^{\prime}$ deletions of the first 951 nucleotides in intron 1 of the $F 13 A 1$ gene, which were inserted into pGL3Basic to produce the constructs. (b) The relative luciferase activity expressed by each construct in U937 cells. * Indicates a statistically significant difference $(P<0.01)$ in the relative luciferase activity between the normal construct pF13AInt1+91 and the mutant construct pF13Alnt1+91mt12. 


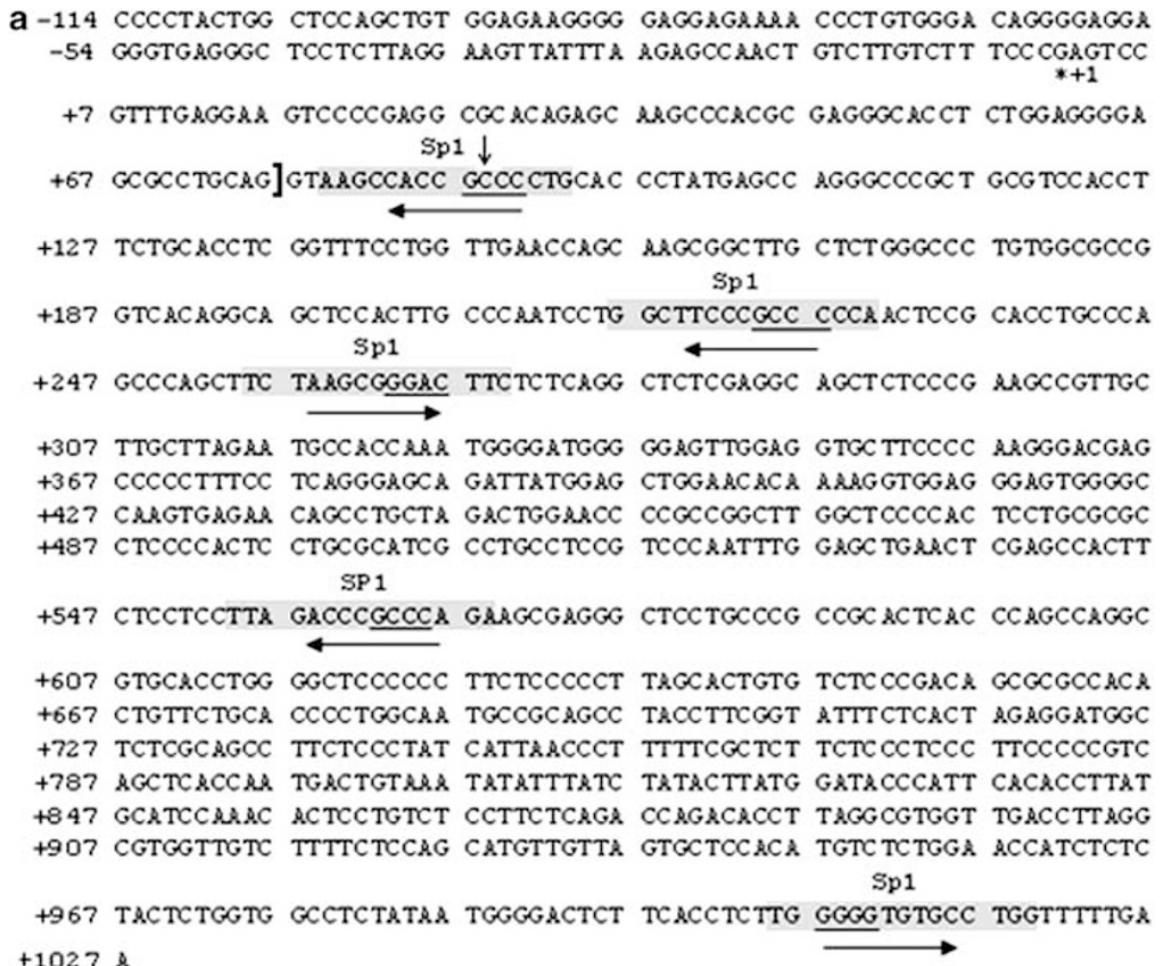

b

d
C

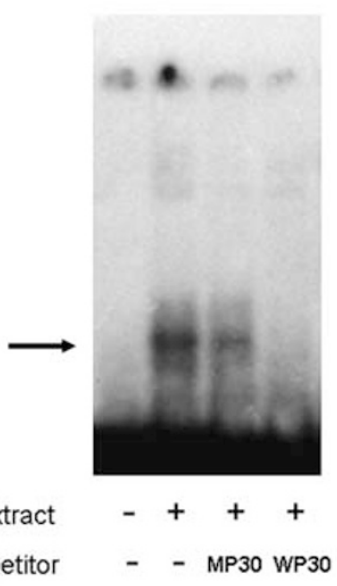

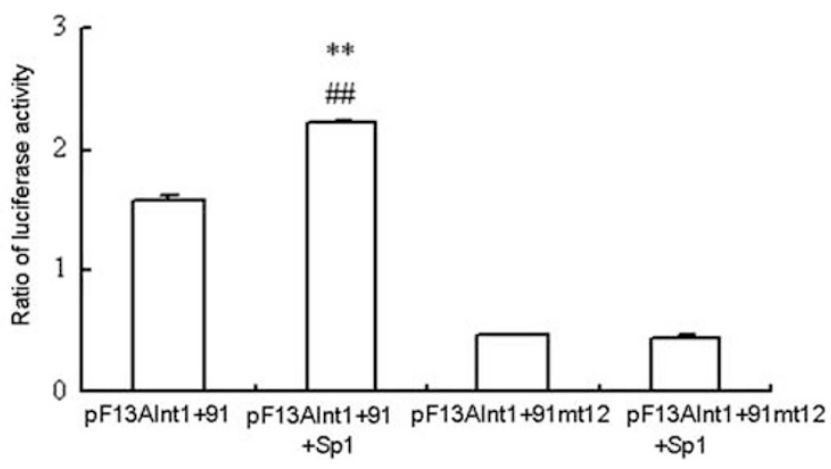

Competition assays showed that the bands were inhibited by both cold wild-type probe and cold mutated probe, although the inhibitory effect of the mutated probe was weaker (Figure 2c). These results suggest that the Int1(+12)A mutation reduces the gene's binding capacity for nuclear protein $\mathrm{Sp} 1$. To determine whether $\mathrm{Sp} 1$ is involved in regulating the expression of the F13A1 gene, the plasmid pN3-Sp1, expressing Sp1, was co-transfected. Compared with the transfection of pF13AInt+91, the co-transfection of pF13AInt+91 and $\mathrm{pN} 3-\mathrm{Sp} 1$ resulted in a 3.78 -fold increase $(P<0.01)$ in luciferase activity in U937 cells (Figure 2d). However, no difference in luciferase 
Figure 2 Sequence from position -114 of the promoter to position +951 of intron 1 of the $F 13 A 1$ gene, electrophoretic mobility shift assay analysis of the sequence at the exon 1/intron 1 border and Sp1 co-transfection analysis. (a) The nucleotide sequence of $114 \mathrm{bp}$ of the minimum promoter, exon 1 , and the first 951 nucleotides at the $5^{\prime}$ end of the first intron. The transcription start site is indicated by an asterisk. Left numbering is relative to the transcription start site. The first exon/intron boundary is separated by a square bracket and the position of Int $1(+12)$ is indicated by a vertical arrow. Five putative binding sites for Sp1 are shaded. The core sequence of the putative Sp1-binding site is underlined. The arrows indicate the directions of the Sp1 sites. (b) Gel-shift analysis of the sequence at the exon 1/intron 1 border covering the $+12 \mathrm{~A}$ mutation hybridized with the wild-type probe (WP30) or the mutant probe (MP30). The arrow indicates the observed gel shifts. (c) Gel-shift analysis of the sequence at the exon 1/intron 1 border in the absence or presence of a 125-fold excess of cold probe. (d) The relative luciferase activities of constructs pF13Alnt1+91 and pF13Alnt1+91mt12 in the absence or presence of a co-transfected construct expressing Sp1. ** Indicates a statistically significant difference $(P<0.01)$ between the cells transfected with pF13AInt1+91 plus $\mathrm{Sp1}$ and pF13AInt1+91. \#\# Indicates that the relative luciferase activity in cells transfected with pF13AInt1+91 plus Sp1 is significantly different from the relative luciferase activity of cells transfected with pF13Alnt $1+91 \mathrm{mt} 12$ or pF13Alnt $1+91 \mathrm{mt} 12$ plus Sp1 $(P<0.01)$.

activity was observed between U937 cells transfected with pF13AInt+91mt12 plus pN3-Sp1 and those transfected with only pF13A+91mt12 (Figure 2d). Therefore, the Sp1-binding site between nucleotides +8 and +15 in intron 1 of the F13A1 gene is involved in regulating the expression of FXIIIA. Therefore, the Int1(+12)A mutation causes the inefficient transcription of F13A1 gene.

\section{CONFLICT OF INTEREST}

The authors declare no conflict of interest.

\section{ACKNOWLEDGEMENTS}

This work was supported by grants from the Natural Science Foundation of China (no. 30060037) and the National Ministry of Education (no. 03147). We thank Professor Akitada Ichinose and Dr Rashida Anwar for their helpful discussions. We also thank the patients and their family for their cooperation and support. We thank Drs DF Zhou, KJ Dong and GQ Zhang for their technical assistance.

1 Karimi, M., Bereczky, Z., Cohan, N. \& Muszbek, L. Factor XIII deficiency. Semin. Thromb. Hemost. 35, 426-438 (2009).

2 Hsieh, L. \& Nugent, D. Factor XIII deficiency. Haemophilia 14, 1190-1200 (2008).

3 Ichinose, A. Physiopathology and regulation of factor XIII. Thromb. Haemost. 86, 57-65 (2001)

4 Anwar, R. \& Miloszewski, K. J. A. Factor XIII deficiency. Br. J. Haematol. 107, 468-484 (1999).
5 Trinh, C. H., Elsayed, W., Eshghi, P., Miri-Moghaddam, E., Zadeh-Vakili, A., Markham, A. F. et al. Molecular analysis of sixteen unrelated factor XIIIA deficient families from south-east of Iran. Br. J. Haematol. 140, 578-592 (2008).

6 Castaman, G., Giacomelli, S. H., Ivaskevicius, V., Schroeder, V., Kohler, H. P., Dragani, A. et al. Molecular characterization of five Italian families with inherited severe factor XIII deficiency. Haemophilia 14, 96-102 (2008).

7 Trigui, N., Frère, C., D’Ercole, C., Chambost, H., Chapuis, N., Pouymayou, C. et al. Molecular characterization of a novel mutation in the factor XIII A subunit gene associated with a severe defect and an adulthood diagnosis. Haemophilia 13, 221-222 (2007).

8 Ariens, R. A. S., Kohler, H. P., Mansfield, M. W. \& Grant, P. J. Subunit antigen and activity levels of blood coagulation factor XIII in healthy individual. Relation to sex, age, smoking and hypertension. Arterioscler. Thromb. Vasc. Biol. 19, 2012-2016 (1999).

9 Kida, M., Souri, M., Yamamotot, M., Saito, H. \& Ichinose, A. Transcriptional regulation of cell type-specific expression of the TATA-less A subunit gene for human coagulation factor XIII. J. Biol. Chem. 274, 6138-6147 (1999).

10 Attié-Castro, F. A., Zago, M. A., Lavinha, J., Elion, J., Rodriguez-Delfin, L., Guerreiro, J. F. et al. Ethnic heterogeneity of the factor XIII Val34Leu polymorphism. Thromb. Haemost. 84, 601-603 (2000).

11 Heng, C. K., Lal, S., Saha, N., Low, P. S. \& Kamboh, M. I. The impact of factor XIIla V34L polymorphism on plasma factor XIII activity in the Chinese and Asian Indians from Singapore. Hum. Genet. 114, 186-191 (2004).

12 Rohrer, J. \& Conley, M. E. Transcriptional regulatory elements within the first intron of Bruton's tyrosine kinase. Blood 91, 214-221 (1998).

13 Shamsher, M. K., Chuzhanova, N. A., Friedman, B., Scopes, D. A., Alhaq, A., Millar, D. S. et al. Identification of an intronic regulatory element in the human protein $\mathrm{C}$ (PROC) gene. Hum. Genet. 107, 458-465 (2000).

14 Warnecke, C., Willich, T., Holzmeister, J., Bottari, S. P., Fleck, E. \& Regitz-Zagrosek, V. Efficient transcription of the human angiotensin II type 2 receptor gene requires intronic sequence elements. Biochem. J. 340, 17-24 (1999).

15 Tanaka, S. \& Ramirez, F. The first intron of the human $\alpha 2(\mathrm{I})$ collagen gene (COL1A2) contains a novel interferon- $\gamma$ responsive element. Matrix. Biol. 26, 185-189 (2007).

16 Girousse, A., Tavernier, G., Tiraby, C., Lichtenstein, L., lacovoni, J. S., Mairal, A. et al. Transcription of the human uncoupling protein 3 gene is governed by a complex interplay between the promoter and intronic sequences. Diabetologia 52, 1638-1646 (2009).

Supplementary Information accompanies the paper on Journal of Human Genetics website (http://www.nature.com/jhg) 